\title{
On the Modular Structure of Local Algebras of Observables
}

\author{
Klaus Fredenhagen * \\ Centre de Physique Théorique, CNRS, Luminy, Case 907, F-13288 Marseille Cedex 9, France
}

\begin{abstract}
An asymptotic expression for the modular operators of local algebras of observables in quantum field theory is given. In an asymptotically scale invariant theory this leads to an identification of the spectra of all modular operators with $\mathbb{R}_{+}$. So in this case the local algebras are algebras of type III, and only factors of type III $_{1}$ can occur in a central decomposition.
\end{abstract}

\section{Introduction}

A characteristic feature of relativistic quantum field theory is the existence of vacuum fluctuations for all local observables. Actually, the vacuum state, restricted to a bounded region $\mathcal{O}$, has many features of an equilibrium state at nonzero temperature. Mathematically, this analogy has been made precise by the Tomita-Takesaki theory [1]. The vacuum vector $\Omega$ is cyclic and separating for each local algebra $\mathfrak{A}(\mathcal{O})$ according to the Reeh-Schlieder theorem [2]. Therefore, the theory of Tomita and Takesaki implies the existence of a positive invertible operator $\Delta_{\mathscr{O}}$ such that $\Delta_{\mathscr{O}}^{i t} \mathfrak{A}(\mathcal{O}) \Delta_{\mathscr{O}}^{-i t} \subset \mathfrak{A}(\mathcal{O})$ for all $t \in \mathbb{R}$, and the vacuum is an equilibrium state with temperature 1 if $\Delta_{\mathscr{O}}^{-i t}$ is considered as the time translation operator.

It is not clear from the beginning whether the "dynamics" which generates these time translations has a physical interpretation. There is an important special case, where such an interpretation is possible. Bisognano and Wichmann [3] have shown that for the wedge $W=\left\{x \in \mathbb{R},\left|x^{0}\right|<x^{1}\right\}$ the operators $\Delta_{w}^{-i t}$ coincide with the unitary operators $U\left(\Lambda_{2 \pi t}\right)$ which implement the Lorentz transformation

$$
\Lambda_{2 \pi t}=\left(\begin{array}{cccc}
\cosh 2 \pi t & \sinh 2 \pi t & & \\
\sinh 2 \pi t & \cosh 2 \pi t & & 0 \\
& & 1 & \\
0 & & & 1
\end{array}\right) .
$$

* Heisenberg fellow 
For an uniformly accelerated observer on the world line $\left\{\Lambda_{2 \pi t}(0, \varrho, 0,0)_{t \in \mathbb{R}}, \varrho>0\right\}$, $\Lambda_{2 \pi \varrho \tau}$ is a translation by proper time $\tau$, hence he finds that the vacuum has temperature $(2 \pi \varrho)^{-1}$, a phenomenon, similar to the Hawking radiation of black holes [4], which has first been discussed by Unruh [5]; the connection with the Bisognano-Wichmann result has been observed by Sewell [6]. Recently, Haag, Narnhofer, and Stein [7] have shown that the Hawking temperature can be explained using only local properties of the theory. They consider the scaling limit of the theory and require that this limit is, in every admissible state and at every point of space time, a Wightman field theory, fulfilling the spectrum condition. Using this property of the scaling limit for points in the intersection of the future and the past horizon (in the case of the accelerated observer considered before the 2-plane $x^{0}=x^{1}=0$ ) they find that the value of the Hawking temperature is uniquely fixed.

These results strongly suggest that the modular operators of finitely extended regions $\mathcal{O}$ might have a physical meaning, too. A purely geometric interpretation of $\Delta_{\mathscr{O}}^{-i t}$, however, seems to be possible only under special conditions [8]. Since $\Delta_{\mathscr{O}}^{-i t}$ induces automorphisms of the algebra of observables and therefore preserves locality, the associated transformation of space time should preserve the causal structure. So only conformal transformations can occur. In fact, Hislop and Longo [9] showed that in the case of the free massless scalar field the modular operator of a double cone induces conformal transformations

$$
\Delta_{\mathscr{O}}^{-i t} \varphi(x) \Delta_{\mathscr{O}}^{i t}=\varphi\left(K_{2 \pi t}\right),
$$

where $\left(K_{t}\right)$ is a 1-parameter subgroup of the conformal group which has a timelike generator and leaves the double cone $\mathcal{O}$ invariant.

To compare this result with that of Bisognano and Wichmann one may look at a double cone $\mathcal{O} \subset W$ which contains the origin in its closure. Then for $x \in \mathcal{O}, x$ sufficiently near to the origin, the actions of $K_{t}$ and $\Lambda_{t}$ approximately coincide,

$$
|x|^{-1}\left(K_{t}-\Lambda_{t}\right) x \rightarrow 0,
$$

where $|x|^{2}=\left(x^{0}\right)^{2}+|x|^{2}$. In the short distance limit, a general theory is expected to exhibit conformal invariance; therefore, one may conjecture that the approximate coincidence of $\Delta_{\mathscr{O}}^{-i t}$ and $U\left(\Lambda_{2 \pi t}\right)$ in the neighbourhood of the origin is a general phenomenon.

Actually, using only the fact that $\Delta_{w}^{-i t} \mathfrak{A}(\lambda \mathcal{O}) \Delta_{w}^{i t} \subset \mathfrak{A}(\mathcal{O})$ for $|t| \leqq(2 \pi)^{-1}|\ln \lambda|, \lambda<1$, which is a consequence of the identification of $\Delta_{w}^{-i t}$ with $U\left(\Lambda_{2 \pi t}\right)$ and the Lorentz covariance of the net of local algebras, one arrives at the following result.

Theorem 1.1. For each $f \in \mathscr{L}_{1}(\mathbb{R})$ and $0<\lambda<1$ there exists a constant $c_{f}(\lambda)>0$ such that:

$$
\begin{gathered}
c_{f}(\lambda) \rightarrow 0 \quad \text { as } \quad \lambda \rightarrow 0, \\
\left\|\int d t f(t)\left\{\Delta_{\mathscr{O}}^{-i t}-\Delta_{w}^{-i t}\right\} A \Omega\right\|^{2} \leqq c_{f}(\lambda)\left\{\|A \Omega\|^{2}+\left\|A^{*} \Omega\right\|^{2}\right\}
\end{gathered}
$$

for all $A \in \mathfrak{H}(\lambda \mathcal{O}) . c_{f}(\lambda)$ neither depends on the details of the theory nor on the size of $\mathcal{O}$. 
The proof of this theorem will be given in Sect. 2 .

We would like to proceed from there to show that the spectra of $\Delta_{\mathscr{Q}}$ and $\Delta_{w}$ coincide. If the theory is dilation invariant this follows simply because in that case we have a group of unitary operators $D(\lambda)$, representing the dilations, with the properties:

$$
D(\lambda) \Omega=\Omega, \quad D(\lambda) \mathfrak{U}(\mathcal{O}) D(\lambda)^{-1}=\mathfrak{U}(\lambda \mathcal{\Theta}), \quad D(\lambda) \Delta_{w}=\Delta_{w} D(\lambda) .
$$

The last relation follows from the fact that the dilations commute with the Lorentz boosts (it would hold even without the identification of the modular transformations with Lorentz boosts since the wedge is dilation invariant). Then

$$
b_{f}(\lambda)=\sup _{A \in \mathscr{2}(\lambda \mathcal{O})}\left\|\int d t f(t) \Delta_{w}^{-i t} A \Omega\right\|\left\{\|A \Omega\|^{2}+\left\|A^{*} \Omega\right\|^{2}\right\}^{-1 / 2} \equiv b_{f}
$$

is independent of $\lambda$. If we choose $f$ such that the support of its Fourier transform $\tilde{f}$ lies in the complement of the spectrum of $\left(-\ln \Delta_{\mathscr{O}}\right)$, then the first term of the lefthand side of inequality (1.5) vanishes, (1.5) reduces to

$$
b_{f}(\lambda)^{2} \leqq c_{f}(\lambda)
$$

for any $\lambda$, and thus by (1.4) $b_{f}=0$ which means that the support of $\tilde{f}$ lies also in the complement of the spectrum of $\left(-\ln \Delta_{w}\right)$. Since the spectrum of $\left(-\ln \Delta_{w}\right)$ is the whole real axis, its complement is empty and thus the spectrum of $\left(-\ln \Delta_{\mathscr{O}}\right)$ must also be the whole real line.

We shall show in Sect. 3 that an analogous argument with the same conclusion can be given if the theory is asymptotically dilation invariant in the following sense: there is a scalar Wightman field $\varphi$ associated to the net of local algebras of observables such that the Wightman functions have a scaling limit.

Can one determine also the spectra of modular operators associated to other normal states of $\mathfrak{H}(\mathcal{O})$ ? This question is related to the type of $\mathfrak{H}(\mathcal{O})$ in the classification of Connes [10]. In a dilation invariant theory it follows immediately from the general results on asymptotically abelian systems $[11,12]$ that the spectra of all modular operators coincide with $\operatorname{sp} \Delta_{\mathfrak{O}}=\mathbb{R}_{+}$. In Sect. 4 we shall show that this remains true under the assumption of asymptotic scale invariance of the Wightman field $\varphi$. In particular, this implies that the local algebras are von Neumann algebras of type III; moreover, in a central decomposition only factors of type III $_{1}$ can occur. In the case of dilation invariant theories this improves a result of Driessler [13].

\section{Comparison of Modular Operators}

Let $\mathscr{N}_{1} \subset \mathscr{N}_{2}$ be von Neumann algebras in a Hilbert space with a common cyclic and separating vector $\Omega$. For $j=1,2, S_{j}$ denotes the closure of the antilinear operator $S_{j}^{(0)}$

$$
S_{j}^{(0)} A \Omega=A^{*} \Omega, \quad A \in \mathscr{N}_{j},
$$

and the modular operator $\Delta_{j}$ is the square of the positive operator occurring in the polar decomposition of $S_{j}$,

$$
S_{j}=J_{j} \Delta_{j}^{1 / 2}
$$


$\mathscr{N}_{1} \subset \mathscr{N}_{2}$ implies $\mathscr{D}\left(S_{1}\right) \subset \mathscr{D}\left(S_{2}\right)$, and from $(2.1)$

$$
S_{2}\left\lceil\mathscr{D}\left(S_{1}\right)=S_{1} .\right.
$$

$\mathscr{D}\left(S_{2}\right)$, equipped with the scalar product

$$
(\Phi, \Psi)_{2}=(\Phi, \Psi)+\left(S_{2} \Psi, S_{2} \Phi\right), \quad \Phi, \Psi \in \mathscr{D}\left(S_{2}\right)
$$

is a Hilbert space which will be denoted by $\mathscr{H}_{2}$, with the identification map $I: \mathscr{H}_{2}$ $\rightarrow \mathscr{D}\left(S_{2}\right) \subset \mathscr{H}$. For $\Phi, \Psi \in \mathscr{D}\left(S_{1}\right)$, from $(2.3)$

$$
(\Phi, \Psi)_{2}=(\Phi, \Psi)+\left(S_{1} \Psi, S_{1} \Phi\right) .
$$

Hence as $S_{1}$ is closed, $\mathscr{H}_{1}=I^{-1} \mathscr{D}\left(S_{1}\right)$ is a closed subspace of $\mathscr{H}_{2}$. Let $P_{1}$ denote the orthogonal projection from $\mathscr{H}_{2}$ onto $\mathscr{H}_{1}$. The operators $V_{j}=I^{-1}\left(1+\Delta_{j}\right)^{-1 / 2}$ are isometries from $\mathscr{H}$ onto $\mathscr{H}_{j}$, with adjoints $V_{j}^{*}=\left(1+\Delta_{j}\right)^{1 / 2} I P_{j}, j=1,2\left(P_{2}=1\right)$. Thus $I V_{j}=\left(1+\Delta_{j}\right)^{-1 / 2}$ and

$$
I P_{j} I^{*}=I V_{j} V_{j}^{*} I^{*}=\left(1+\Delta_{j}\right)^{-1}, \quad j=1,2 .
$$

Equation (2.6) implies in particular that for an increasing sequence of von Neumann algebras $\mathscr{N}^{(n)}, \mathscr{N}_{1} \subset \mathscr{N}^{(n)} \subset \mathcal{N}_{2},\left\{\cup \mathscr{N}^{(n)}\right\}^{\prime \prime}=\mathscr{N}_{2}$, the modular operators converge in the sense of strong resolvent convergence [14]. We shall use Eq. (2.6) to estimate the difference

$$
\left[\left(1+\Delta_{2}\right)^{-1}-\left(1+\Delta_{1}\right)^{-1}\right] A \Omega, \quad A \in \mathscr{N}_{1},
$$

by the time spent by $A$ in $\mathscr{N}_{1}$ under the time evolution corresponding to $\Delta_{2}$.

Proposition 2.1. Let $A \in \mathscr{N}_{1}$ such that $\Delta_{2}^{i t} A \Delta_{2}^{-i t} \in \mathscr{N}_{1}$ for $|t| \leqq \tau$. Then

$$
\left\|\left[\left(1+\Delta_{2}\right)^{-1}-\left(1+\Delta_{1}\right)^{-1}\right] A \Omega\right\| \leqq \frac{2}{\pi} \tanh ^{-1} e^{-\pi \tau}\left\{\|A \Omega\|^{2}+\left\|A^{*} \Omega\right\|^{2}\right\}^{1 / 2} .
$$

Proof. From (2.6) $I^{*}=I^{-1}\left(1+\Delta_{2}\right)^{-1}$. We have

$$
I^{-1}\left(1+\Delta_{2}\right)^{-1}=s-\lim _{\varepsilon \succ 0} I^{-1} \Delta_{2}^{\varepsilon}\left(1+\Delta_{2}\right)^{-1}=s-\lim _{\varepsilon \succ 0} I^{-1} \int d t f_{\varepsilon}(t) \Delta_{2}^{i t}
$$

with $f_{\varepsilon}(t)=\frac{i}{2}[\sinh (t+i \varepsilon) \pi]^{-1}$, and therefore,

$$
\begin{aligned}
{\left[\left(1+\Delta_{2}\right)^{-1}-\left(1+\Delta_{1}\right)^{-1}\right] A \Omega } & =I\left(1-P_{1}\right) I^{-1}\left(1+\Delta_{2}\right)^{-1} A \Omega \\
& =s-\lim \int d t f_{\varepsilon}(t) I\left(1-P_{1}\right) I^{-1} \Delta_{2}^{i t} A \Omega .
\end{aligned}
$$

For $|t| \leqq \tau$ one has $\Delta_{2}^{i t} A \Delta_{2}^{-i t} \in \mathscr{N}_{1}$, hence $\Delta_{2}^{i t} A \Omega \in \mathscr{D}\left(S_{1}\right), I^{-1} \Delta_{2}^{i t} A \Omega \in \mathscr{H}_{1}$ and $\left(1-P_{1}\right) I^{-1} \Delta_{2}^{i t} A \Omega=0$. Thus one can restrict the $t$-integration in (*) to values $|t|>\tau$. The limit $\varepsilon>0$ can then be performed under the integral sign, and the proposition follows with $\left\|I^{-1} \Delta_{2}^{i t} A \Omega\right\|=\left\{\|A \Omega\|^{2}+\left\|A^{*} \Omega\right\|^{2}\right\}^{1 / 2}$ for all $t$ and $\int_{|t| \geqq \tau} d t \frac{1}{2}|\sinh \pi t|^{-1}$ $=\frac{2}{\pi} \tanh ^{-1} e^{-\pi \tau}$. q.e.d. 
If one replaces $\Delta_{j}$ by $e^{u} \Delta_{j}$ in Proposition $2.1, j=1,2, u \in \mathbb{R}$, one obtains, by the same method, the estimate

$$
\left.\|\left[1+e^{u} \Delta_{2}\right)^{-1}-\left(1+e^{u} \Delta_{1}\right)^{-1}\right] A \Omega \| \leqq \frac{2}{\pi} \tanh ^{-1} e^{-\pi \tau}\left\{\|A \Omega\|^{2}+e^{u}\left\|A^{*} \Omega\right\|^{2}\right\}^{1 / 2} .
$$

The next lemma gives a connection between the resolvants $\left(1+e^{u} \Delta\right)^{-1}$ and the unitaries $\Delta^{i t}$ for a positive invertible operator $\Delta$.

Lemma 2.2. Let $\varphi \in \mathscr{S}(\mathbb{R})$ with $\int \varphi(u) d u=0$. Then

$$
\int d u \varphi(u)\left(1+e^{u} \Delta\right)^{-1}=\int d t \frac{i}{2}[\sinh \pi t]^{-1} \tilde{\varphi}(t) \Delta^{i t}
$$

with $\tilde{\varphi}(t)=\int d u \varphi(u) e^{i t u}$.

Proof. Using the spectral representation of $\ln \Delta$, the lemma follows from the string of identities

$$
\begin{aligned}
\int d t & \frac{i}{2}[\sinh \pi t]^{-1} \tilde{\varphi}(t) e^{i x t} \\
& =\int d t \int d u \frac{i}{2}[\sinh \pi t]^{-1} \varphi(u) e^{i t(x+u)} \\
& =\lim _{\varepsilon>0} \int d u \varphi(u) \int d t \frac{i}{2}[\sinh \pi(t+i \varepsilon)]^{-1} e^{i t(x+u)} \\
& =\lim _{\varepsilon>0} \int d u \varphi(u) e^{\varepsilon(x+u)}\left(1+e^{x+u}\right)^{-1} \\
& =\int d u \varphi(u)\left(1+e^{x+u}\right)^{-1} . \quad \text { q.e.d. }
\end{aligned}
$$

Now we have the means to prove Theorem 1.1.

Proof of Theorem 1.1. Let $\mathfrak{U}(\mathcal{O})=\mathscr{N}_{1}, \mathfrak{U}(W)=\mathcal{N}_{2}, \Delta_{\mathcal{O}}=\Delta_{1}$, and $\Delta_{w}=\Delta_{2}$. For $g \in \mathscr{D}(\mathbb{R})$, we define

$$
\varphi_{g}(u)=(2 \pi)^{-1} \int d \operatorname{tg}(t)(-2 i) \sinh (\pi t) e^{-i t u} .
$$

We have the estimates

$$
\begin{array}{rl}
\| \int d & d f(t)\left(\Delta_{2}^{i t}-\Delta_{1}^{i t}\right) A \Omega \| \\
\leqq & \int d t|f(t)-g(t)| \cdot 2\|A \Omega\| \\
& \quad+\int d u\left|\varphi_{g}(u)\right|\left\|\left[\left(1+e^{u} \Delta_{2}\right)^{-1}-\left(1+e^{u} \Delta_{1}\right)^{-1}\right] A \Omega\right\| \\
\leqq & \left\{\int d t|f(t)-g(t)|+\int_{|u| \geqq c} d u\left|\varphi_{g}(u)\right|\right\} \cdot 2\|A \Omega\| \\
& +\int d u\left|\varphi_{g}(u)\right| e^{c / 2} \frac{2}{\pi} \tanh ^{-1} \lambda^{1 / 2}\left\{\|A \Omega\|^{2}+\left\|A^{*} \Omega\right\|^{2}\right\}^{1 / 2},
\end{array}
$$

which give the theorem with

$$
c_{f}(\lambda)=\inf _{\substack{g \in \mathscr{D}(\mathbb{R}) \\ c>0}}\left\{2 \int d t|f-g|+2 \int_{|u| \geqq c} d u\left|\varphi_{g}\right|+\frac{2}{\pi} \tanh ^{-1} \lambda^{1 / 2} e^{c / 2} \int d u\left|\varphi_{g}\right|\right\} \text {. q.e.d. }
$$




\section{The Spectrum of $\Delta_{\mathfrak{o}}$}

For a determination of $\operatorname{sp} \Delta_{\mathfrak{O}}$ by Theorem 1.1 in a dilationally invariant theory one needs some information on the quantity $b_{f}(\lambda)$ defined in Eq. (1.7). In a certain sense the local algebras $\mathfrak{I}(\lambda \mathcal{O})$ must be large enough for arbitrarily small $\lambda$. We, therefore, restrict ourselves to asymptotically dilation invariant theories. Since there is no satisfying intrinsic definition of asymptotic dilation invariance available, we use the following characterization: there exists a scalar hermitian Wightman field $\varphi$ which is associated to the local algebras of observables such that the closure $\varphi(f)^{-}$of $\varphi f$ ) is affiliated to $\mathfrak{A}(\mathcal{O})$ if supp $f \subset \mathcal{O}$. Furthermore, there exists a monotone function $N: \mathbb{R}_{+}^{*} \rightarrow \mathbb{R}_{+}^{*}$ such that the scaled Wightman functions $W_{n}^{\lambda}$,

$$
W_{n}^{\lambda}\left(x_{1}, \ldots, x_{n}\right)=N(\lambda)^{n}\left(\Omega, \varphi\left(\lambda x_{1}\right) \ldots \varphi\left(\lambda x_{n}\right) \Omega\right),
$$

converge for $\lambda \rightarrow 0$ in the sense of tempered distributions with $\lim W_{2}^{\lambda} \equiv W_{2}^{0}$ different from zero. Such a behaviour is expected for renormalizable theories with an ultraviolet fixed point.

It is convenient to smear the field $\varphi$ only over trajectories $\left\{\Lambda_{t} x\right\}$ with $x \in W$. According to a result of Borchers [16] this is sufficient to get well defined operators. We introduce the operators

$$
\varphi_{\varrho}(g)=N(\varrho) \int d t g(t) \varphi(\varrho \sinh 2 \pi t, \varrho \cosh 2 \pi t, 0,0),
$$

with $\varrho>0$ and $g \in \mathscr{D}(\mathbb{R})$. If $\operatorname{supp} g \subset(-L, L)$ then $\varphi_{\varrho}(g)^{-}$is affiliated to $\mathfrak{A}\left(\mathcal{O}_{R}\right)$, $R=\varrho e^{L}$, where $\mathcal{O}_{R}, R \in \mathbb{R}_{+}$, denotes the double cone with center $(0, R, 0,0)$ and radius $R$. $\varphi_{\varrho}$ may be considered as a one-dimensional field which transforms covariantly under the Lorentz transformations $\Lambda_{t}$,

$$
U\left(\Lambda_{2 \pi t}\right) \varphi_{\varrho}(\tau) U\left(\Lambda_{2 \pi t}\right)^{-1}=\varphi_{\varrho}(\tau+t) .
$$

The Fourier transforms of the two-point functions of these fields

$$
\left\{\int d t e^{-i k \tau}\left(\Omega, \varphi_{\varrho}(0) \varphi_{\varrho}(\tau) \Omega\right)\right\} d k=d v_{\varrho}(k)
$$

are positive measures. They are tempered, as a consequence of the temperedness of the field $\varphi$; their support is the real line; this follows for instance by a representation as an integral over mass values with the Kallen-Lehmann weight $\mu$ of the two-point function of the field $\varphi$,

$$
d v_{\varrho}(k)=\left\{\int d \mu\left(m^{2}\right) B_{\varrho m}(k)\right\} d k(N(\varrho) / \varrho)^{2},
$$

where $B_{o m}(k)$ is an analytic function with nonnegative values for $m \geqq 0^{1}$; furthermore, $d v_{\varrho}(-k)=e^{-k} d v_{\varrho}(k)$; this is the expression of the fact that $\Omega$ induces a KMS-state on the field $\varphi_{\varrho}$ with temperature 1 and implies in particular that multiplication by $e^{-k}$ does not destroy the temperedness of $v_{\varrho}$; and finally, from the scaling assumption on $\varphi$, the measures converge for $\varrho \rightarrow 0$ in the sense of tempered distributions to a tempered measure $v_{0}$ which has the same properties.

The fields $\varphi_{e}$ can be used for an investigation of the function $b_{f}(\lambda)$. Let $f \in \mathscr{L}_{1}(\mathbb{R})$ and $g \in \mathscr{D}(\mathbb{R})$. Then for $\lambda>0$ and $\varrho$ sufficiently small $\varphi_{\varrho}(g)^{-}$is affiliated

1 Explicitly, $B_{m}(k)=2 m^{2}\left\{K_{1+i k / 2 \pi}(m) K_{1-i k / 2 \pi}(m)-K_{i k / 2 \pi}(m) K_{-i k / 2 \pi}(m)\right\}$ for $m \neq 0$, where $K_{n}$ denotes the modified Bessel function of the third kind of order $n$ [17] 
to $\mathfrak{A}(\lambda \mathcal{O})$, hence

$$
b_{f}(\lambda) \geqq\left\|\int d t f(t) \Delta_{w}^{-i t} \varphi_{\varrho}(g) \Omega\right\|^{2}\left\{\left\|\varphi_{\varrho}(g) \Omega\right\|^{2}+\left\|\varphi_{\varrho}(g)^{*} \Omega\right\|^{2}\right\}^{-1} .
$$

Because of the Lorentz covariance of the field $\varphi_{\varrho}(3.3)$ and the KMS-property of the measure $v_{\varrho}$, the right-hand side of inequality (3.6) is equal to

$$
\frac{\int\left\{d v_{\varrho}(k)+d v_{\varrho}(-k)\right\}|\tilde{g}(k)|^{2}|\tilde{f}(k)|^{2}\left(1+e^{-k}\right)^{-1}}{\int\left\{d v_{\varrho}(k)+d v_{\varrho}(-k)\right\}|\tilde{g}(k)|^{2}} .
$$

In the limit $\varrho \rightarrow 0$ the support restriction for $g$ disappears. So varying $g$ freely in $\mathscr{D}(\mathbb{R})$, one finds that the lowest upper bound of the term in (3.7) is $\sup \left\{|\tilde{f}(k)|^{2}\left(1+e^{-k}\right)^{-1}, \quad k \in \mathbb{R}\right\}$. This number, however, is equal to $\left\|\int d t f(t) \Delta_{w}^{-i t}\left(1+\Delta_{w}\right)^{-1 / 2}\right\|^{2}$, which is an upper bound for the right-hand side of inequality (3.6), since from the definition of the modular operator

$$
\left\|\int d t f(t) \Delta_{w}^{-i t}\left(1+\Delta_{w}\right)^{-1 / 2}\right\|^{2}=\sup _{A \in \mathfrak{N}(W)} \frac{\left\|\int d t f(t) \Delta_{w}^{-i t} A \Omega\right\|^{2}}{\|A \Omega\|^{2}+\left\|A^{*} \Omega\right\|^{2}} .
$$

Hence $b_{f}(\lambda)$ is independent of $\lambda$ also in the case of asymptotic dilation invariance, and one concludes that $\operatorname{sp} \Delta_{\mathscr{O}}=\mathbb{R}_{+}$, in the same way as in the dilation invariant case.

\section{Spectra of all Modular Operators}

There are general theorems asserting that the spectra of all modular operators coincide for asymptotically abelian systems $[11,12]$. In a Wightman field theory the scale transformations of the fields act in a similar way as the automorphisms in an asymptotically abelian system; in fact, in a dilation invariant theory the analogy is exact. The intuitive reason for the independence of the spectrum of the modular operator from the choice of the normal faithful state is that the unitary cocycles relating different modular automorphism groups approximately commute with operators $A \in \mathfrak{A}(\lambda \Theta)$ for $\lambda$ sufficiently small; only these operators have been used to identify $\operatorname{sp} \Delta_{\mathscr{O}}$.

It is not known whether the general structure of quantum field theory implies that the center of $\mathfrak{A}(\mathcal{O})$ is always contained in the center of the whole algebra of observables [this is known to be the case e.g. for the algebra $\mathfrak{U}(W)$ ]. Actually, Buchholz [18] has given simple examples of local nets, where the local algebras have nontrivial centers, whereas the center of the global algebra is trivial; these examples have all properties usually required with the exception of duality and the time slice axiom.

Therefore, it is reasonable to consider also the modular operators associated to normal states which are not necessarily faithful. If $\omega$ is a normal state of a von Neumann algebra $\mathscr{N}$ there is a minimal projection $E \in \mathscr{N}$ with $\omega(E)=1$. The restriction $\omega_{E}$ of $\omega$ to the reduced algebra $\mathscr{N}_{E}=E \mathscr{N} E$ is faithful, so one can introduce the modular operator $\Delta_{\omega}$ as the modular operator associated to the pair $\left(\omega_{E}, \mathscr{N}_{E}\right)$. The intersection

$$
S^{\prime}(\mathscr{N})=\cap \operatorname{sp} \Delta_{\omega}
$$


where $\omega$ ranges over the set of normal states of $\mathscr{N}$ is an algebraic invariant of $\mathscr{N}$ [19]. It coincides with the invariant $S$ of Connes [10] if $\mathscr{N}$ is a factor.

For the determination of $\operatorname{sp} \Delta_{\omega}$ we use the following criterion which is a slight variation of a criterion of Stormer [12].

Proposition 4.1. Let $\omega$ denote a normal state of a von Neumann algebra $\mathscr{N}$, and let $\lambda>0$. Then $\lambda \in \operatorname{sp} \Delta_{\omega}$ iff for each $\varepsilon>0$ there is some $A \in \mathscr{N}$ with $\omega\left(A^{*} A\right) \geqq 1$ such that for all $B \in \mathscr{N}$,

$$
|\omega(A B)-\lambda \omega(B A)| \leqq \varepsilon\left\{\omega\left(B^{*} B\right)+\lambda \omega\left(B B^{*}\right)\right\}^{1 / 2} .
$$

Proof. Let $\mathscr{N}$ act on a Hilbert space $\mathscr{H}$ such that $\omega$ is induced by the cyclic vector $\Omega$. Let $E$ denote the minimal projection in $\mathcal{N}$ with $\omega(E)=1$. If $\lambda \in \operatorname{sp} \Delta_{\omega}$ there exists, for each $\varepsilon>0$, some $A \in \mathscr{N}_{E}$ with $\|A \Omega\|=1$ and $\left\|\left(\Delta_{\omega}^{1 / 2}-\lambda^{1 / 2}\right) A \Omega\right\|<\varepsilon / \sqrt{2}$. Then for all $B \in \mathscr{N}$,

$$
\begin{aligned}
|\omega(A B)-\lambda \omega(B A)| & \left.=\mid\left(\Delta_{\omega}^{1 / 2}-\lambda^{1 / 2}\right) A \Omega,\left(\Delta_{\omega}^{1 / 2}+\lambda^{1 / 2}\right) E B^{*} \Omega\right) \mid \\
& \leqq \frac{\varepsilon}{\sqrt{2}}\left\|\left(\Delta_{\omega}^{1 / 2}+\lambda^{1 / 2}\right) E B^{*} \Omega\right\| \\
& \leqq \varepsilon\left\{\left\|\Delta_{\omega}^{1 / 2} E B^{*} \Omega\right\|^{2}+\lambda\left\|E B^{*} \Omega\right\|^{2}\right\}^{1 / 2} \\
& =\varepsilon\left\{\|E B \Omega\|^{2}+\lambda\left\|E B^{*} \Omega\right\|^{2}\right\}^{1 / 2} \\
& \leqq \varepsilon\left\{\omega\left(B^{*} B\right)+\lambda \omega\left(B B^{*}\right)\right\}^{1 / 2} .
\end{aligned}
$$

If, on the other hand, for some $\varepsilon>0$ there exists an $A \in \mathscr{N}$ with $\omega\left(A^{*} A\right) \geqq 1$ and $|\omega(A B)-\lambda \omega(B A)| \leqq \varepsilon\left\{\omega\left(B^{*} B\right)+\lambda \omega\left(B B^{*}\right)\right\}^{1 / 2}$ for all $B \in \mathcal{N}$, then for $B \in \bar{N}_{E}$

$$
\begin{aligned}
\left|\left(\left(\Delta_{\omega}^{1 / 2}-\lambda^{1 / 2}\right) E A \Omega,\left(\Delta_{\omega}^{1 / 2}+\lambda^{1 / 2}\right) B^{*} \Omega\right)\right| & \leqq \varepsilon\left\{\left\|\Delta_{\omega}^{1 / 2} B^{*} \Omega\right\|^{2}+\lambda\left\|B^{*} \Omega\right\|^{2}\right\}^{1 / 2} \\
& \leqq \varepsilon\left\|\left(\Delta_{\omega}^{1 / 2}+\lambda^{1 / 2}\right) B^{*} \Omega\right\|
\end{aligned}
$$

and therefore, $\left\|\left(\Delta_{\omega}^{1 / 2}-\lambda^{1 / 2}\right) E A \Omega\right\| \leqq \varepsilon$. The norm of $E A \Omega$ can be estimated by inserting $B=A^{*}(1-E)$ into the criterion. Because of $(1-E) \Omega=0$, this gives

$$
\lambda\|(1-E) A \Omega\|^{2} \leqq \varepsilon \lambda^{1 / 2}\|(1-E) A \Omega\|
$$

and thus $\|(1-E) A \Omega\| \leqq \varepsilon \lambda^{-1 / 2}$ and $\|E A \Omega\|^{2} \geqq 1-\varepsilon^{2} \lambda^{-1}$. For $\varepsilon<\lambda^{1 / 2}$, one gets

$$
\operatorname{dist}\left(\operatorname{sp} \Delta_{\omega}^{1 / 2}, \lambda^{1 / 2}\right) \leqq \frac{\varepsilon}{\left(1-\varepsilon^{2} \lambda^{-1}\right)^{1 / 2}}
$$

so if the criterion is fulfilled for all $\varepsilon>0$ one finds $\lambda \in \operatorname{sp} \Delta_{\omega}$. q.e.d.

Theorem 4.2. Let $\mathcal{N}$ be a von Neumann algebra with $\mathfrak{U}(\mathcal{O}) \subset \mathscr{N} \subset \mathfrak{U}(W)$. If a Wightman field $\varphi$ with a scale invariant limit is associated to the local net then $S^{\prime}(\mathcal{N})=\mathbb{R}_{+}$.

Proof. We shall use the Wightman field $\varphi$ to construct, for each $\lambda>0, \varepsilon>0$, a uniformly bounded sequence $A_{n} \in \mathfrak{U}\left(\frac{1}{n} \mathcal{O}\right)$ such that

$$
\left\|A_{n} \Omega\right\| \geqq 1 \quad \text { and } \quad\left\|\left(j\left(A_{n}^{*}\right)-\lambda^{1 / 2} A_{n}\right) \Omega\right\| \leqq \varepsilon
$$


for all $n \in \mathbb{N}$, where $j$ denotes the modular involution of $(\mathfrak{A}(W), \Omega)$, i.e. $j$ is the PCTtransformation composed with a rotation by $\pi$ in the $x_{2}-x_{3}$-plane.

Provided these sequences exist, the argument goes as follows. Let $\omega$ be a normal state of $\mathscr{N}$, and let $\Phi \in \mathscr{H}$ be a vector inducing $\omega$. ( $\Phi$ exists since $\mathscr{N}$ has a separating vector; the argument below, however, works equally well with density matrices.) Since the weak limit points of the sequences $\left(\left|A_{n}\right|^{2}\right)$ and $\left(\mid j\left(A_{n}^{*}-\left.\lambda^{1 / 2} A_{n}\right|^{2}\right.\right.$ are localized in the origin they are multiples of the identity; hence for some $n$, $\left\|A_{n} \Phi\right\| \geqq 1$ and $\left\|\left(j\left(A_{n}^{*}\right)-\lambda^{1 / 2} A_{n}\right) \Phi\right\|,\left\|\left(A_{n}^{*}-\lambda^{1 / 2} j\left(A_{n}\right)\right) \Phi\right\| \leqq \varepsilon$. But then the criterion of Proposition 4.1 is satisfied:

$$
\begin{aligned}
\left|\omega\left(A_{n} B\right)-\lambda \omega\left(B A_{n}\right)\right|= & \mid\left(\left[A_{n}^{*}-\lambda^{1 / 2} j\left(A_{n}\right)\right] \Phi, B \Phi\right)+\lambda^{1 / 2}\left(j\left(A_{n}\right) \Phi, B \Phi\right) \\
& -\lambda^{1 / 2}\left(B^{*} \Phi,\left[\lambda^{1 / 2} A_{n}-j\left(A_{n}^{*}\right)\right] \Phi\right)-\lambda^{1 / 2}\left(B^{*} \Phi, j\left(A_{n}^{*}\right) \Phi\right) \mid \\
\leqq & \varepsilon\left\{\|B \Phi\|+\lambda^{1 / 2}\left\|B^{*} \Phi\right\|\right\} \\
\leqq & \varepsilon \sqrt{2}\left\{\omega\left(B^{*} B\right)+\lambda \omega\left(B B^{*}\right)\right\}^{1 / 2} .
\end{aligned}
$$

It remains to establish the existence of the sequences $\left(A_{n}\right)$. We choose

$$
A_{n}=\left[1+c \varphi_{1 / n+k}(f)^{-} \varphi_{1 / n+k}(f)^{*}\right]^{-1} \varphi_{1 / n+k}(f)^{-}
$$

with $f \in \mathscr{D}(\mathbb{R}), c>0$ and $k \in \mathbb{N}$ such that the Fourier transform of $f$ is concentrated around $\ln \lambda, A_{n} \in \mathfrak{U}\left(\frac{1}{n} \mathcal{O}\right)$ and $c$ is sufficiently small. More precisely, we choose $f$ such that $\left\|\varphi_{1 / n}(f) \Omega\right\| \geqq 1+\varepsilon / 3$ and $\|\left(\Delta_{W}^{1 / 2}-\lambda^{1 / 2)} \varphi_{1 / n}(f) \Omega \| \leqq \varepsilon / 3\right.$ for all sufficiently large $n$. This is possible since the measures $\left(1+e^{-k}\right) v_{\varrho}$ are tempered and converge for $\varrho \rightarrow 0$. The constant $c$ will be chosen such that $\left(1+\lambda^{1 / 2}\right)\left\|\left[A_{n}-\varphi_{1 / n}(f)\right] \Omega\right\|$ and $\left\|\Delta_{w}^{1 / 2}\left(A_{n}-\varphi_{1 / n}(f)\right) \Omega\right\|$ are bounded by $\varepsilon / 3$. This is possible uniformly in $n$ because of the estimates

$$
\begin{gathered}
\left\|\left(A_{n}-\varphi_{1 / n}(f)\right) \Omega\right\| \leqq(\sqrt{c} / 2)\left\|\varphi_{1 / n}(f)^{*} \varphi_{1 / n}(f) \Omega\right\| \\
\left\|\Delta_{w}^{1 / 2}\left(A_{n}-\varphi_{1 / n}(f)\right) \Omega\right\| \leqq(\sqrt{c} / 2)\left\|\varphi_{1 / n}(f) \varphi_{1 / n}(f)^{*} \Omega\right\|,
\end{gathered}
$$

and the assumed scaling properties of the 4-point function. Since $\Delta_{w}^{1 / 2} A_{n} \Omega$ $=j\left(A_{n}^{*}\right) \Omega$, the sequence $\left(A_{n}\right)$ has the properties required above. q.e.d.

If a von Neumann algebra $\mathscr{N}$ with $\mathfrak{A}(\mathcal{O}) \subset \mathscr{N} \subset \mathfrak{U}(W)$ is a factor, then the Connes invariant $S(\mathcal{N})[10]$ coincides with $S^{\prime}(\mathscr{N})$, which is equal to $\mathbb{R}_{+}$according to Theorem 4.2. Hence $\mathscr{N}$ is a factor of type III $_{1}$. Moreover, if $E$ is a projection in the center of $\mathscr{N}$ such that $\mathscr{N}_{E}=E \mathscr{N} E$ is a factor, one has $S\left(\mathscr{N}_{E}\right)=S^{\prime}\left(\mathscr{N}_{E}\right)$ $\supset S^{\prime}(\mathscr{N})=\mathbb{R}_{+}$and finds that $\mathscr{N}_{E}$ is a factor of type $\mathrm{III}_{1}$. An analogous statement holds for factors occurring in a direct integral central decomposition of $\mathscr{N}$.

Proposition 4.3. Let $\mathscr{N}$ be a von Neumann algebra in a separable Hilbert space. If the invariant $S^{\prime}(\mathcal{N})$ defined in Eq. (4.1) is equal to $\mathbb{R}_{+}$, only type $I I I_{1}$-factors can occur in the central decomposition of $\mathscr{N}$.

Proof. Let $\left(\sigma_{t}\right)$ denote the modular automorphism group associated to a faithful normal state of $\mathscr{N},(\mathscr{N} \underset{\sigma}{X} R)$ the crossed product of $\mathscr{N}$ by $\sigma$, and $\Theta$ the dual action of $\mathbb{R}$ on the crossed product. Then by a theorem of Connes and Takesaki [20]

$$
e^{s} \in S^{\prime}(\mathscr{N}) \Leftrightarrow \Theta_{s} \text { acts trivially on the center of }(\mathscr{N} \underset{\sigma}{X} \mathbb{R}) \text {. }
$$


Now let $\mathscr{N}=\int^{\oplus} \mathscr{N}(r) d \mu(r)$ denote the central decomposition of $\mathscr{N}$. According to Sutherland [21] there are corresponding direct decompositions of the modular automorphisms, $\sigma_{t}=\int^{\oplus} \sigma_{t}^{(r)} d \mu(r)$, of the crossed product, of the center of the crossed product and of the dual action, $\Theta_{s}=\int^{\oplus} \Theta_{s}^{(r)} d \mu(r) . S^{\prime}(\mathscr{N})=\mathbb{R}_{+}$implies then that, for all $s \in \mathbb{R}, \Theta_{s}^{(r)}$ is trivial on the center of $\left(\mathscr{N}(r) \underset{\sigma^{(r)}}{\mathbf{X}} \mathbb{R}\right)$ for $\mu$-almost every $r$. Hence, for $\mu$-almost every $r, \Theta_{s}^{(r)}$ is trivial on the center of $\left(\mathcal{N}(r) \underset{\sigma^{(r)}}{X} \mathbb{R}\right)$ for all $s \in \mathbb{Q}$ and, because of continuity, for all $s \in \mathbb{R}$. Thus $S^{\prime}(\mathcal{N}(r))=\mathbb{R}_{+}$, i.e. $\mathscr{N}(r)$ is a type $\mathrm{III}_{1^{-}}$ factor for $\mu$-almost every $r$. q.e.d.

The results in this section show in particular that there exist no intermediate type I factors between $\mathfrak{A}(\mathcal{O})$ and $\mathfrak{A}(W)$ in contrast to the situations considered recently by Doplicher and Longo [22]. The physical reason is that the commutant $\mathfrak{U}(W)^{\prime}$ of $\mathfrak{A}(W)$ is the algebra associated to the left wedge $W^{\prime}$ which has a common boundary point with $\mathcal{O}$. In such a situation product states, i.e. states without correlations between measurements in $W^{\prime}$ and $\mathcal{O}$, cannot exist, a fact which was known from the work of Roberts in the dilation invariant case [23].

Acknowledgements. The ideas for this work arose from discussions with Rudolf Haag and Daniel Kastler on Ref. [7]. These discussions are gratefully acknowledged. I also would like to thank Detlev Buchholz, Joachim Cuntz, Daniel Kastler, and Roberto Longo for valuable suggestions on the meaning of the invariant $S^{\prime}$ which finally led to the proof of Proposition 4.3, Rudolf Haag for useful hints concerning the presentation of the results and Daniel Kastler for the hospitality extended to me at the Centre de Physique Théorique of the C.N.R.S., Luminy.

\section{References}

1. Takesaki, M.: Tomita's theory of modular Hilbert algebras. Berlin, Heidelberg, New York: Springer 1970

2. Reeh, H., Schlieder, S.: Bemerkungen zur Unitäräquivalenz von Lorentzinvarianten Feldern. Nuovo Cimento 22, 1051 (1961)

See also: Streater, R.F., Wightman, A.S.: PCT, spin and statistics and all that. New York: Benjamin 1964

3. Bisognano, J.J., Wichmann, E.H.: On the duality for a hermitian scalar field. J. Math. Phys. 16, 985 (1975)

4. Hawking, S.W.: Particle creation by black holes. Commun. Math. Phys. 43, 199 (1975)

5. Unruh, W.G.: Notes on black-hole evaporation. Phys. Rev. D 14, 870 (1976)

6. Sewell, G.L.: Relativity of temperature and the Hawking effect. Phys. Lett. 79 A, 23 (1980)

7. Haag, R., Narnhofer, H., Stein, U.: On quantum field theory in gravitational background. Commun. Math. Phys. 94, 219-238 (1984)

8. Haag, R.: Private communication

9. Hislop, P.D., Longo, R.: Modular structure of the local algebras associated with the free massless scalar field theory. Commun. Math. Phys. 84, 71 (1982)

10. Connes, A.: Une classification des facteurs de type III. Ann. Ec. Norm. Sup. 6, 133 (1973)

11. Araki, H.: Remarks on spectra of modular operators of von Neumann algebras. Commun. Math. Phys. 28, 267 (1972)

12. St $\varnothing$ rmer, E.: Spectra of states and asymptotically abelian $C^{*}$-algebras. Commun. Math. Phys. 28, 279 (1972) [corrections Commun. Math. Phys. 38, 341 (1974)] 
13. Driessler, W.: On the type of local algebras in quantum field theory. Commun. Math. Phys. 53, 295 (1977)

14. Longo, R.: A simple proof of the existence of modular automorphisms in approximately finite dimensional von Neumann algebras. Pac. J. Math. 75, 199 (1978) and private communication

15. Longo, R.: Algebraic and modular structure of von Neumann algebras of physics. Proc. Symp. Pure Math. 38, Part 2, 551 (1982)

16. Borchers, H.-J.: Field operators as $C^{\infty}$-functions in spacelike directions. Nuovo Cimento 38, 1600 (1964)

17. Erdelyi, A., Magnus, W., Oberhettinger, F.: Higher transcendental functions. New York, Toronto, London: McGraw-Hill 1953

18. Buchholz, D.: Private communication

19. Pedersen, G.K.: $C^{*}$-algebras and their automorphism groups, LMS Monographs 14. London, New York: Academic Press 1979

20. Connes, A., Takesaki, M.A.: The flow of weights on factors of type III. Tôhoku Math. J. 29, 473 (1977)

21. Sutherland, C.: Crossed products, direct integrals, and Connes' classification of type III factors. Math. Scand. 40, 209 (1977)

22. Doplicher, S.: Local aspects of superselection rules. I. Commun. Math. Phys. 85, 73 (1982) Doplicher, S., Longo, R.: Local aspects of superselection rules. II. Commun. Math. Phys. 88, 399 (1983)

23. Roberts, J.E.: Some applications of dilatation invariance to structural questions in the theory of local observables. Commun. Math. Phys. 37, 273 (1974)

Communicated by G. Mack

Received April 26, 1984 
